\title{
Le Festin de pierre (II, 1, 413-479 ; III, 1, 894-938) de Thomas Corneille
}

Traduction (de Giancarlo Peris) et note à la traduction

\section{Annamaria Laserra}

\section{(2) OpenEdition}

\section{Journals}

Édition électronique

URL : http://journals.openedition.org/rief/289

DOI : $10.4000 /$ rief.289

ISSN : 2240-7456

Éditeur

Seminario di filologia francese

Référence électronique

Annamaria Laserra, «Le Festin de pierre (II, 1, 413-479 ; III, 1, 894-938) de Thomas Corneille », Revue italienne d'études françaises [En ligne], 3 | 2013, mis en ligne le 15 décembre 2013, consulté le 22 septembre 2020. URL : http://journals.openedition.org/rief/289 ; DOI : https://doi.org/10.4000/rief.289

Ce document a été généré automatiquement le 22 septembre 2020.

\section{c) (i) $९$}

Les contenus de la RIEF sont mis à disposition selon les termes de la Licence Creative Commons Attribution - Pas d'Utilisation Commerciale - Pas de Modification 4.0 International. 


\section{Le Festin de pierre (II, 1, 413-479 ; III, 1, 894-938) de Thomas Corneille}

Traduction (de Giancarlo Peris) et note à la traduction

Annamaria Laserra

\section{RÉFÉRENCE}

Le Festin de pierre (1683), II, 1, 413-479 ; III, 1, 894-938, dans Molière, CEuvres complètes, éd. G. Forestier, Paris, Gallimard, « Bibliothèque de la Pléiade », vol. II, 2010, p. 1259-1260 et 1274-1275

Je remercie les éditions Euno de Leonforte pour m'avoir autorisée à publier ici un extrait de la traduction de Th. Corneille, Le Festin de pierre, édité et préfacé par A. Laserra et traduit par G. Peris (sous presse).

\section{Version originale}

ACTE II

Scène Première

CHARLOTTE, PIERROT

CHARLOTTE

Nostre-dinse, Piarrot, pour les tirer de peine,

Tu t'es là rencontré bien à point.

PIERROT

Oh, marguenne,

Sans nou ç'en était fait. 415

CHARLOTTE

Je le crois bian.

PIERROT

Vois-tu, 
Il ne s'en fallait pas l'époisseur d'un fétu,

Tous deux de se nayer eussiont fait la sottise.

CHARLOTTE

C'est donc l'vent da matin...

PIERROT

Aga quien, sans feintise,

Je te vas tout fin drait conter par le menu,

Comme en n'y pensant pas le hasard est venu. 420

Il aviont bien besoin d'un œil comme le nôtre,

Qui les vît de tout loin, car c'est moi, com's dit l'autre,

Qui les ai le premier avisés. Tanquia don,

Sur le bord de la Mar bien leu prend que j'équion,

Où de tarre Gros-Jean me jetait une motte, 425

Tout en batifolant, car com' tu sais, Charlotte,

Pour v'nir batifoler Gros-Jean ne charche qu'ou,

Et moi parfouas aussi je batifole itou.

En batifolant don, j'ai fait l'apercevance

D'un grouillement su gliau, sans voir la différence, 430

De squi pouvait grouiller; ça grouillait à tous coups,

Et grouillant, par secousse allait comme envars nous.

J'estas embarrassé, s'n'était point stratagème,

Et tout com' je te vois, je voyas ça de même,

Aussi fixiblement, et pis tout d'un coup, quien, 435

Je voyas qu'après ça je ne voyas plus rien.

Eh, Gros-Jean, ç'ai-je fait, stanpendant que je somme

À niaiser parmi nous, je pens' que vla de zhomme,

Qui nagiant tout là-bas. Bon, sm'a-t-il fait, vrament,

T'auras de queuque Chat vu le trépassement ; 440

T'as la vu' trouble. Oh bian, ç'ai-je fait, t'as biau dire,

Je n'ai point la vu' trouble, et sn'est point jeu pour rire,

C'est la de zomme. Point, sm'a-t-il fait, sn'en est pas,

Piarrot, t'as la barlue. Oh! j'ai sque tu voudras,

Ç'ai-je fait, mais gageon que j'n'ai point la barlue, 445

Et qu'ça qu'en voit là-bas, ç'ai-je fait, qui remue,

C'est de zomme, vois-tu, qui nageont vars ici.

Gag' que non, sm'a-t-il fait. Oh margué, gag' que si,

Dix sols. Oh, sm'a-t-il fait, je le veux bian, marguenne;

Quien, mets argent su jeu; vla le mien. Palsanguenne 450

Je n'ai fait là-dessous l'Étourdi ni le Fou,

J'ai bravement bouté par tarre mes dix sou,

Quatre pièces tapées et le restant en double,

Jarnigué, jé verron si j'avon la vu' trouble.

Ç'ai-je fait, les boutant... plus hardiment enfin 455

Que si j'eusse avalé queuque varre de Vin ;

Car je sis hasardeux moi, qu'en m'mette en boutade,

Je vas sans tant d'raisons tout à la débandade.

Je savas bian pourtant sque j'faisas d'en par là,

Queuque gniais! Enfin don, j'n'on pas putôt mis, vla, 460 
Que j'voyon tout à plein com' deu Zomme à la nage Nous faision signe ; et moi, sans rien dir davantage, De prendre les enjeux. Allon, Gros-Jean, allon,

Ç'ai-je fait, vois-tu pas comme ils nous appellon?

Ils s'vont s'nayer. Tant mieux, sm'a-t-il fait, je m'en gausse, 465

Ils m'ant fait pardre. A donc le tirant par lé chausse,

J'l'ai si bien sarmonné, qu'à la parfin vars eux, J'avon dans une Barque avironné tous deux.

Et pis cahin caha, j'on tant fait que je somme

Venus tout contre, et pis j'les avon tiré comme 470

Il aviont quasi bu déjà pu que de jeu,

Et pis j'les on cheu nous menés auprès du feu,

Où je l'zon vu tout nus sécher leu Zoupelande,

Et pis il en venu deux autres de leur bande.

Qui s'équian, vois-tu bian, sauvez tout seul, et pis 475

Mathurine est venue à voir leu biaux Habits ;

Et pis ils liont conté qu'al n'était pas tant sotte,

Qu'al avait du malin dans l'œil, et pis, Charlotte,

Vla tout com'ça s'est fait pour te l'dire en un mot.

[...]

ACTE III

Scène Première

SGANARELLE

[...]

Que croyez-vous?

DON JUAN

Je crois ce qu'il faut que je croie.

SGANARELLE

Bon, parlons doucement, et sans vous échauffer. 895

Le Ciel?

DON JUAN

Laissons cela.

SGANARELLE

C'est fort bien dit. L'Enfer?

DON JUAN

Laissons cela, te dis-je !

SGANARELLE

Il n'est pas nécessaire

De vous expliquer mieux, votre réponse est claire.

Malheur si l'esprit fort s'y trouvait oublié.

Voilà ce que vous sert d'avoir étudié, 900

Temps perdu. Quant à moi, personne ne peut dire

Que l'on m'ait rien appris, je sais à peine lire ;

Et j'ai de l'ignorance à fond ; mais franchement,

Avec mon petit sens, mon petit jugement,

Je vois, je comprends mieux ce que je dois comprendre. 905

Que vos livres jamais ne pourraient me l'apprendre.

Ce monde où je me trouve, et ce Soleil qui luit, 
Sont-ce des Champignons venus en une nuit?

Se sont-ils faits tout seuls? Cette masse de pierre,

Qui s'élève en Rochers, ces Arbres, cette Terre, 910

Ce Ciel planté là-haut, est-ce que tout cela

S'est bâti de soi-même ? Et vous, seriez-vous là,

Sans votre Père à qui le sien fut nécessaire,

Pour devenir le vôtre ? Ainsi, de Père en Père,

Allant jusqu'au premier, qui veut-on qui l'ait fait, 915

Ce premier? Et dans l'Homme, Ouvrage si parfait ;

Tous ces os agencés l'un dans l'autre, cette âme,

Ces veines, ce poumon, ce cœur, ce foie... Oh Dame,

Parlez à votre tour comme les autres font,

Je ne puis disputer si l'on ne m'interrompt ; 920

Vous vous taisez exprès, et c'est belle malice.

DON JUAN

Ton raisonnement charme, et j'attends qu'il finisse.

SGANARELLE

Mon raisonnement est, Monsieur, quoi qu'il en soit, Que l'Homme est admirable en tout, et qu'on y voit

Certains ingrédients, que plus on les contemple, 925

Moins on peut expliquer. D'où vient que... par exemple,

N'est-il pas merveilleux que je suis ici, moi,

Et qu'en la tête, là, j'aie un je ne sais quoi,

Qui fait qu'en un moment, sans en savoir la cause,

Je pense, s'il le faut, cent différentes choses, 930

Et ne me mêle point d'ajuster les ressorts

Que ce je ne sais quoi fait mouvoir dans mon corps,

Je veux lever un doigt, deux, trois, la main entière, Aller à droite, à gauche, en avant, en arrière...

DON JUAN, apercevant Léonor :

Ah, Sganarelle, vois. Peut-on sans s'étonner... 935

SGANARELLE

Voilà ce qu'il vous faut, Monsieur, pour raisonner.

Vous n'êtes point muet en voyant une Belle.

DON JUAN

Celle-ci me ravit.

SGANARELLE

Vraiment.

DON JUAN

Que cherche-t-elle?

SGANARELLE

Vous devriez déjà l'être allé demander.

\section{Traduction italienne}

ATTO II

Scena Prima

CARLOTTA, PIERO 
CARLOTTA

Porca paletta, Pie', pe' metteli a l'asciutto, Ce sei arrivato a ciccio.

PIERO

Pe' la miseria! un lutto,

Senza de noi, de certo, s'era verificato. 415

CARLOTTA

Ce credo, Pie', ce credo.

PIERO

E invece hanno svortato,

Ma ce bastava poco : pe'n torsolo spolpato

Pe' 'na malappa, e quelli stavino a sangozza'.

CARLOTTA

Er vento de mattina...

PIERO

Senza gnente inguatta',

Te ricconto preciso quello che ch'adè successo,

Come, senza pensacce, er destino s'è espresso ; 420

Ci avevino bisogno de 'n occhio come er mio,

Ché, come ha detto l'antro, l'ho visti proprio io,

Che l'ho visti pe' primo. Perciò, allora, n'è vero ?

Me ne stavo lì ar Pirgo, pe' fortuna che c'ero,

Quanno Gianni er ciccione, me butta rena in testa, 425

Lo conosci, Carlo', ci ha la mano assai lesta,

Je piace ar buzzicone svagasse co 'sto gioco,

De le vorte a me pure me piace, e manco poco.

Giocanno a 'sta maniera vedo, nun so perché,

Un movimento a mare : 'n se capisce ch'adè 430

Da che se stava a move; ce stava un cavallone,

Che annava verso er molo, faceva assai impressione

Ero fori de testa, nun me raccapezzavo,

E come mo te guardo, io allora lo guardavo,

Fisso e come affatato, ma dopo a l'improviso, 435

Nun se vedeva gnente; e io serio e deciso

Je dissi : «A Ci', n'campana! Ner mentre che noi adesso

Stamo qui a cazzeggià, uno sta là malmesso

Là in fonno sta a nota' ! ... allora lui m'ha fatto :

" A Pie', ma che stai a di', starà a affugasse un gatto, 440

Nun ci hai la vista bona »; " Ma sei scemo - j'ho detto -

La vista ce l'ho bona, so' certo e nun so' gretto,

Là ce stanno de l'òmmini ». E lui : " macché, 'n c'è gnente.

A Pie', ci hai le visioni "... « Va be' sarò un veggente.

Ma scommettemo, ho detto, che quella 'n è visione. 445

Quello che ho visto in fonno, j'ho detto, e 'n c'è questione,

So' omi, guarda un po', che vonno veni' qui ... »

«Scommettemo de no, m'ha fatto ... Va be', sì ;

«Mortacci, dieci sordi ! ... m'ha fatto, me li gioco ;

«Scommetti ? Ecco li mia! Che possi prenne foco ! » 450 
Io nun ho fatto er sordo, e manco ho fatto er matto

E je buttai per terra li sordi, a quer coatto,

Quattro scudi stampati, er resto de bajocchi,

«Porca troia vedemo, si ce l'ho boni l'occhi!»

J'ho fatto io, buttannoli pe' terra, un po' arterato 455

Come si pe' 'n goccetto adero frastornato;

Pe' via che so azzarda', si me pii pe' 'r culo,

Manno tutto pe' l'aria, a carci, come un mulo ;

Sapevo che facevo ar molo co' lui appresso,

«E che te credi? A l'urtimo, dopo d'ave' scommesso, 460

Avemo visto due che stavino a nota',

J'avemo fatto segno e io, senza parla',

So' subito partito, annamo Ciccio, annamo,

Vedi come ce chiamino? Mejo si se sbrigamo.

Stanno a affuga'... Adè mejo, m'ha fatto, e che me frega ? 465

Pe' loro ho perso ». E io, « basta mo' co' sta bega »,

Allora l'ho agguantato, strillato e l'ho convinto,

E avemo preso un vuzzo, indove che l'ho spinto,

E poi daje a rema', a fadica' de brutto,

A tiralli di fori, ero guasi distrutto $; 470$

Avevino bevuto, assai più che pe' gioco

E dopo l'ho portati da noi, davanti ar foco,

'Ndove l'ho visti 'gnudi, a asciugasse i vestiti,

E quindi so' arrivati antri e due intirizziti,

Gente de quela ghenga che s'erino sarvati, 475

Po' arriva Maturina, vede i panni lavati,

E loro a dije che nun è mica una goja,

E che ci ha l'occhio tenero. E po', Carlotta, gioia,

Ecco ch'adè successo, a di' co' 'na parola.

[...]

ATTO III

Scena Prima

SGANARELLO

[...]

In che cosa credete?

DON GIOVANNI

Io credo in ciò che devo.

SGANARELLO

Bene. Parliamo piano, senza litigio eterno 895

Il Cielo?

DON GIOVANNI

E dai, finiamola...

SGANARELLO

Sì, ben detto ! l'Inferno ?

DON GIOVANNI

Finiamola, ti dico.

SGANARELLO

Non dovete per niente 
Spiegarvi ancora meglio. La risposta è evidente. Guai se il pensiero forte fosse dimenticato Ecco a che cosa serve l'avere assai studiato ; 900 Tempo perso. Per me non c'è chi si dia pena Di avermi addottrinato : io so legger appena ; La mia ignoranza è grande ; ma vedo francamente, Col mio poco buon senso, senz'arguzia di mente, Che a volte capto meglio ciò che c'è da carpire, 905 Di quanto i vostri libri mi potranno mai dire. Il mondo in cui mi trovo, questo sole lucente Sono forse dei funghi germogliati dal niente? Si son fatti da soli ? Questa pietra ammassata Che s'eleva in scogliere ; questa terra infiorata, 910 Questo Cielo piantato lassù dove conviene Si costruì da sé ? E voi, voi stesso, ebbene, Vivreste senza il padre al quale il suo servì Per diventare il vostro ? dal padre in giù così, Retrocedendo al primo, mi chiedo quale mente 915 Lo fece. E il corpo umano, quest'opera eccellente Queste ossa strutturate, per non dir delle anime Le vene, e i polmoni, e gli occhi, ma che diamine! Ma parlate anche voi al modo di altra gente! Non riesco a discutere se non c'è un contendente ; 920 Fate apposta a tacere, voi siete molto astuto. DON GIOVANNI

Continua, è affascinante, attento sarò, e muto. SGANARELLO

Il mio ragionamento, alla fine, Signore, È che l'uomo è ammirevole, e nel mondo interiore, Vedi certi ingredienti che, più li stai a guardare, 925 Meno li puoi spiegare. Come mai... non vi pare? Non è meraviglioso che io sia qui, proprio io, E che nel capo... qui... io abbia un non so ... Oddio! Che fa che in un momento, ne ignoro le cagioni, Penso, se necessario, a cento e più ragioni, 930 E non mi curo affatto di aggiustar gli ingranaggi Che oscure forze muovono senza mandar messaggi? Io voglio alzare un dito, due, tre, la mano intera, Andare a destra, a manca, su e giù, mattina e sera... DON GIOVANNI, scorgendo Eleonora :

Ah, Sganarello, guarda, si può senza stupirsi... 935

SGANARELLO

Ecco cosa vi serve per ragionare e dirsi.

Voi non restate muto davanti a una bella DON GIOVANNI

Questa qui mi rapisce. SGANARELLO

Toh. 
DON GIOVANNI

Cosa cerca, ella?

SGANARELLO

Dovreste essere andato già a chiederle ragione.

\section{Note à la traduction}

1 La mise en scène, en 1665, de Dom Juan ou le Festin de pierre, comédie en prose de Molière, souleva de telles accusations d'immoralité que son auteur la retira après quinze représentations. La pièce réapparut après la mort de l'écrivain, dans une version révisée dans la forme (désormais en vers) et dans le contenu (n'y figuraient plus les scènes controversées - que Molière avait du reste lui-même en partie coupées lors des premières représentations). Pendant deux siècles, on ne connut Le Festin que dans cette version, dont l'auteur, Thomas Corneille (frère cadet de Pierre), passait en son temps comme l'un des meilleurs versificateurs de France. Puis on revint au texte en prose de Molière, et celui de Thomas Corneille fut oublié. Il conserve pourtant un intérêt non seulement lié à sa versification, parfois remarquable, mais, avec le recul, à sa dimension documentaire et historique, qui permet de mieux saisir comment certaines préoccupations esthétiques et morales du XVII ${ }^{e}$ siècle ont pesé sur le Dom Juan de Molière.

2 La pièce de Thomas Corneille étant née pour être en vers par rapport à un original en prose, il a semblé nécessaire de lui laisser sa spécificité en italien, en donnant une traduction en vers rimés. Giancarlo Peris a recouru au vers dont la mesure parait adaptée à la traduction de l'alexandrin français, le martelliano (composé de deux hémistiches d'un heptasyllabe chacun), qu'il a rendu dans toute la gamme de ses accentuations italiennes : tronquées (tronche), pleines (piane) ou coulantes (sdrucciole).

3 Pour donner un aperçu le plus représentatif possible de ce qu'ont été le travail de réécriture en vers de Thomas Corneille et cette présente traduction en italien, j'ai choisi deux extraits, une scène en dialecte (II, 1), l'autre en français standard (III, 1).

Les protagonistes sont, dans les deux cas, des gens du peuple. Des paysans, dans le premier extrait; Sganarelle dans l'autre. Le langage des paysans est un patois proche de celui de l'île-de-France, tel qu'on le parlait aux alentours de la capitale au XVII ${ }^{\mathrm{e}}$ siècle ; celui de Sganarelle est un français courant à l'époque. Dans le premier cas, l'effet comique est lié à l'emploi populaire de la langue par les personnages; dans le second, aux à-peu-près de Sganarelle, incapable d'adapter sa rhétorique aux idées qu'il voudrait défendre face à son maître.

5 Pour les passages en dialecte, Giancarlo Peris a eu l'idée de créer une correspondance géographique en recourant au civitavecchiese parlé à Civitavecchia, unique variante du romanesco romain. Choix heureux, me semble-t-il. Civitavecchia se trouvant en bord de mer, la localisation de la scène du naufrage y apparaitt parfaitement légitimée. Peris a d'ailleurs accentué la couleur locale par une allusion au Pirgo, la plage préférée des habitants de cette ville. Il traduit le vers par lequel débute le récit de Pierrot : «Sur le bord de la mar, bian leu prend que j'équion » (qui littéralement se dirait en italien : In riva al mare, buon per loro che ci fossi) en localisant la scène : « Me ne stavo lì ar Pirgo, pe' fortuna che c'ero » (vers 424). Des mots (et des expressions) civitavecchiesi font écho 
aux termes et expressions dialectales du texte français - par exemple "malappa » (voile extérieur de l'oignon) pour « fétu ».

6 Parodié par Molière à travers une altération graphique des mots donnant une caricature phonétique du langage paysan, le texte présente les mêmes déformations dans la version de Thomas Corneille, alors que, l'italien étant une langue tonique (piana), où les syllabes sont toutes entièrement prononcées, aucune modification orthographique n'a été nécessaire pour rendre ce langage. C'est sur d'autres registres que la traduction italienne renvoie au français. On notera certaines modifications dialectales concernant des éléments lexicaux similaires. C'est le cas des prénoms (toujours prononcé Piarrot en français, Pierrot est par exemple souvent apocopé en "Pie"» en italien), des sobriquets ("Gros Jean» devient "Gianni er ciccione", maintenant inaltérée l'ironie sur le défaut physique ciblé par le français), ou des imprécations et des jurons : faute de mots ou expressions parfaitement correspondants dans les deux langues, Giancarlo Peris a essayé d'en maintenir la « couleur ». Prenons le cas de l'expression par laquelle débute le texte (« Nostre-dinse » pour Notre-Dame): Charlotte adoucit un blasphème jusqu'à le renverser pour exprimer son admiration devant l'entreprise de Pierrot. « Nostre-dinse, Piarrot ! devient alors « Porca paletta, Pie'! ( (expression dont la signification dans un italien moins populaire serait rendue par un bien plus faible "accidenti!», en général utilisée à la place de jurons plus marqués). Les différents « marguenne ! », " palsanguenne », prononcés par Gros Jean au moment où il perd un pari de « dix sols » (« dieci sordi») sont exprimés en italien par un expressif « Mortacci!»

7 Le jargon dialectal utilisé par Molière et Thomas Corneille présente toute une série d'altérations émaillant le texte. Des suppressions ("v'nir » pour " venir », " pis » pour «puis»), des condensations («sugliau» pour «sur l'eau»). Elles peuvent affecter la conjugaison, les accords des participes, l'exactitude des désinences et celle des pronoms personnels et possessifs, comme «Il aviont bien besoin ». De même, le jargon utilisé dans le texte italien foisonne d'un type analogue d'altérations. Des suppressions (ex. «quela » pour " quella»: "Gente de quela ghenga »), des substitutions ("de le vorte » pour «delle volte »); des modifications affectant des pronoms : "gli» devient « je » ( «j'ho detto», «j'ho fatto»), les thèmes des verbes («Te ricconto preciso»), plus rarement les désinences (« Ci avevino bisogno »).

8 Le registre du premier texte est énonciatif et parodique. Énonciatif : Pierrot rapporte comment il a sauvé Don Juan d'une noyade. Parodique : l'« effet paysan » se manifeste à travers le recours à une orthographe phonétique, apte, non seulement à imiter la prononciation des protagonistes (ex : «C'est la de zomme » / "C'est là des hommes »), mais aussi à souligner, par la mise en évidence d'« impropriétés » grammaticales, les approximations linguistiques paysannes (utilisation incorrecte $\mathrm{du}$ substantif « homme » au singulier; du partitif; fusion de la marque de liaison - « $\mathrm{z}$ » pour « $\mathrm{s}$ »intégrée au mot « zomme », exaltant l'effet comique de cet énoncé).

9 Le registre du deuxième extrait est argumentatif et parodique à la fois. Essayant de contrecarrer l'athéisme de Don Juan, Sganarelle se lance dans une longue tirade où il aborde de façon très mal assurée l'argument de la cause première. N'arrivant pas à porter à terme son raisonnement, il s'en prend à son maître, qu'il accuse de ne pas intervenir, le laissant, ainsi, dans l'embarras. Une comparaison entre les versions françaises et la traduction italienne de ce passage donnera une idée des solutions adoptées par Peris. Chez Molière, à Sganarelle qui s'exclame: «[...] Vous vous taisez 
exprès, et me laissez parler par belle malice ", Don Juan réplique : "J'attends que ton raisonnement soit fini ». Thomas Corneille reprend presque littéralement son modèle : "Vous vous taisez exprès, et c'est belle malice », dit son Sganarelle. La réponse de Don Juan renforce l'ironie de l'original: "Ton raisonnement charme, et j'attends qu'il finisse ». Le choix de Peris est d'accentuer ce ton moqueur. À Sganarelle qui récrimine : «Fate apposta a tacere, voi siete molto astuto", Don Juan retorque: "Continua, è affascinante, attento sarò, e muto ». Par ce mot ajouté en fin de vers, Peris suit Thomas Corneille en montrant un Don Juan ironique, qui se dit attentif et fasciné, mais met aussi en exergue son impassibilité face aux contorsions rhétoriques de son serviteur. L'embarras de Sganarelle apparaît alors sans issue, face au mutisme de Don Juan ; un mutisme qui, exprimé par cet adjectif «muto » qu'une virgule isole des deux autres, apparaît irrévocable et, si l'on peut dire, presque sculpté.

INDEX

Mots-clés : traduction, vers classique, Festin de pierre, dialecte, alexandrin, martelliano, Corneille (Thomas) 